\title{
GOVERNANÇA DE DADOS APLICADA À CIÊNCIA DA INFORMAÇÃO: ANÁLISE DE UM SISTEMA DE DADOS CIENTÍFICOS PARA A ÁREA DA SAÚDE
}

\author{
DATA GOVERNANCE APPLIED TO INFORMATION SCIENCE: ANALYSIS OF A \\ SCIENTIFIC DATA SYSTEM FOR THE HEALTH AREA
}

GOBERNANZA DE DATOS APLICADA A LA CIENCIA DE LA INFORMACIÓN: ANÁLISIS DE UN SISTEMA DE DATOS CIENTÍFICOS PARA EL ÁREA DE LA SALUD

Priscilla Lüdtke Espíndola ${ }^{1}$

José Francisco Salm Junior ${ }^{1}$

Francisco Rosa ${ }^{1}$

Jordan Paulesky Juliani ${ }^{1}$

${ }^{1}$ Universidade do Estado de Santa Catarina

Correspondência

${ }^{1}$ Priscilla Lüdtke Espíndola (D)

Universidade do Estado de Santa Catarina

Florianópolis, SC

E-mail: priscillaludtke@gmail.com

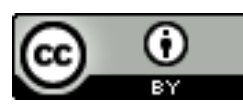

JITA: LN. Data base management systems.

Submetido em: $27 / 11 / 2017$

Aceito em: 01/08/2018

Publicado em: 16/08/2018 
RESUMO: Os dados são insumos valiosos para as organizações e para estudos científicos. Para que os dados científicos possam ser empregados em estudos relacionados à Avaliação Motora, e produzirem resultados confiáveis, é necessária a adoção de boas práticas de coleta, armazenamento e recuperação. Esta pesquisa tem como objetivo aplicar o "Modelo de ciclo de vida dos dados" e as ferramentas de governança de dados para identificar oportunidades de melhorias para o Sistema de Avaliação Motora, especialmente com relação a qualidade de dados. Quanto aos aspectos metodológicos, o estudo é caracterizado como uma pesquisa aplicada com caráter exploratório, coleta de dados realizada por meio de pesquisa-ação, e análise dos dados por meio de métodos qualitativos. Como resultados, constatou-se a necessidade de redesenhar o sistema estudado, incluindo mecanismo para tratamento dos dados para evitar duplicidade e garantir homogeneidade e completude. Igualmente, verificou-se ser necessário criar e implementar uma política para restringir que somente profissionais da saúde e da educação aptos possam inserir dados no Sistema. Compreende-se que as boas práticas da governança de dados, princípios do "Modelo de ciclo de vida dos dados" e demais ferramentas adotadas neste estudo contribuíram para diagnosticar falhas e identificar oportunidades de melhoria no Sistema de Avaliação Motora.

PAlavraS-Chave: Governança de dados. Ciclo de Vida dos Dados. Dados Científicos. Dados em saúde. Qualidade de dados.

ABSTRACT: Data are valuable inputs to organizations and to scientific studies. In order for scientific data to be used in studies related to Motor Assessment, and to produce reliable results, good collection, storage and retrieval practices are required. This research aims to apply "Data Life Cycle Model" data governance tools to identify opportunities for improvements to the Motor Assessment System, especially related to data quality. Regarding to the methodological aspects, the study is characterized as a applied research with an exploratory feature, data collection performed through research-action, and data analysis through qualitative methods. As results, it was verified the need to redesign the studied system, including mechanism for data treatment to avoid duplicity and guarantee homogeneity and completeness. Likewise, it was found necessary to create and implement a policy to restrict that only able health and education professionals could enter data in the System. It is understood that good data governance practices, "Data Life Cycle Model" principles and other tools adopted in this study contributed to diagnose failures and identify opportunities for improvement in the Motor Assessment System.

KEYWORDS: Data governance. Data Life Cycle. Scientific Data. Health data. Data quality.

RESUMEN: Los datos son aportaciones valiosas para las organizaciones y para los estudios científicos. Para que los datos científicos se utilicen en estudios relacionados con la evaluación motora y para producir resultados confiables, se requieren buenas prácticas de recolección, almacenamiento y recuperación. Esta investigación tiene como objetivo aplicar herramientas de gobernanza de datos del "Modelo de ciclo de vida de los datos" para identificar oportunidades de mejoras en el Sistema de Evaluación Motor, especialmente en relación con la calidad de los datos. En cuanto a los aspectos metodológicos, el estudio se caracteriza como una investigación aplicada con una característica exploratoria, la recolección de datos realizada a través de la investigación-acción, y el análisis de datos a través de métodos cualitativos. Como resultado, se verificó la necesidad de rediseñar el sistema estudiado, incluido el mecanismo de tratamiento de datos para evitar la duplicidad y garantizar la homogeneidad y la integridad. Del mismo modo, se consideró necesario crear e implementar una política para restringir que solo los profesionales de salud y educación capaces pudieran ingresar datos en el Sistema. Se entiende que las buenas prácticas de gobernanza de datos, los principios del "Modelo de ciclo de vida de los datos" y otras herramientas adoptadas en este estudio contribuyeron a diagnosticar fallas e identificar oportunidades de mejora en el Sistema de Evaluación Motor.

PAlabras Clave: Gobernanza de datos. Ciclo de vida de los datos. Datos científicos. Datos de salud. Calidad de datos. 


\section{INTRODUÇÃ̃o}

Atualmente, estamos permeados por dados e informações em diversas atividades cotidianas. Os dados e as informações possuem um papel fundamental para a vida em sociedade, pois é por meio deles que podemos embasar nossas decisões, conhecer melhor o mundo que nos cerca, bem como adquirir conhecimento.

No contexto corporativo, a maneira que os dados são gerenciados pode influenciar a agilidade, a produtividade, os custos e o tempo necessários para atender às solicitações de clientes internos e externos. Além disso, os dados possuem um importante papel na prestação de contas nas organizações com seus stakeholders e com a sociedade em geral.

A governança de dados é entendida por Santos (2010) como uma ação multidisciplinar que tem como finalidade tratar os dados como insumos ativos e tangíveis nas organizações. Para isso, a governança de dados determina políticas, padronizações, processos, papéis, responsabilidades e tecnologias para melhor acompanhar e monitorar os dados gerados, armazenados, utilizados e eliminados na organização.

Ainda segundo a autora a ausência de qualidade dos dados é um inibidor de sucesso nas organizações. O controle da qualidade dos dados nas organizações é um dos processos compreendidos pela governança de dados. Por meio da governança de dados são definidas métricas, procedimentos e requisitos que auxiliam a organização a atingir a qualidade de dados necessária para cumprir suas demandas e alcançar seus objetivos (BARATA, 2015).

Entende-se que dados de qualidade também são essenciais para o sucesso de pesquisas científicas, pois os dados científicos requerem a adoção de boas práticas de coleta, armazenamento e recuperação.

Na visão de Corrêa (2016) os dados científicos possuem um papel de destaque no avanço da produção científica, e por esse motivo, atualmente, vem demandando novas ações para garantir sua preservação e sua recuperação.

Nesse contexto, compreende-se que os conceitos e boas práticas determinadas pela governança de dados em organizações, podem igualmente serem aplicados à sistemas de informação que realizam a coleta, armazenamento e recuperação de dados científicos.

Tendo em vista a importância do processo de ciclo de vida dos dados em pesquisas científicas, sobretudo na área da Ciência da Informação (CI), Santana (2013) realizou uma análise de Modelos de Ciclo de Vida de Dados desenvolvidos por diversas instituições e autores. Os referidos Modelos embasaram a identificação de quatro fases que representam o comportamento e o fluxo da informação, dentro do contexto da CI: coleta, armazenamento, 
recuperação e descarte, bem como seis objetivos que permeiam tais fases: privacidade, qualidade, direitos autorais, integração, disseminação e preservação.

A junção dessas quatro fases, permeadas pelos seis objetivos, foi denominada pelo autor de Modelo de ciclo de vida dos dados para Ciência da Informação (CVD-CI), o qual possui como objetivo ser um modelo de ciclo de vida de dados que considere as características e especificidades da CI.

Na visão de Santana e Bonini (2014), por meio de estudos e pesquisas acerca do ciclo de vida de dados, a CI pode ampliar a democratização do uso dos dados, contribuir com a divulgação científica, viabilizar o acesso aos dados e, consequente aos conhecimentos gerados por meio da aplicação desses dados.

Desse modo, diante da importância de dados de qualidade em pesquisas científicas e a necessidade de adoção de um processo de ciclo de vida com etapas definidas, que podem proporcionar mais segurança para o pesquisador no manejo dos dados, têm-se a seguinte pergunta de pesquisa: como o CVD-CI e ferramentas de diagnóstico e de governança de dados podem ser empregadas na elaboração de uma proposta de melhorias para o Sistema de Avaliação Motora (SAM)?

O SAM é um sistema on-line para inserção dos dados de avaliação motora de crianças e idosos, o qual é utilizado por Profissionais da Saúde e da Educação (PSE) para auxiliar na aplicação do método de avaliação motora baseado na Escala de Desenvolvimento Motor (EDM). Por meio do SAM, os PSE podem registrar os dados de avaliação motora de cada paciente, consultar o histórico das avaliações realizadas, e também acompanhar a evolução das intervenções realizadas em seus pacientes (SISTEMA..., [201-?]). Trata-se, portanto, de um sistema com uma base de dados aberta, voltada para o desenvolvimento de pesquisas científicas, definição de políticas públicas e tratamento de pacientes, para os públicos acadêmico, político e PSE respectivamente.

Nesse contexto, este estudo tem como objetivo geral: aplicar o CVD-CI e as ferramentas de governança de dados para identificar oportunidades de melhorias para o SAM especialmente com relação a qualidade de dados. E como objetivos específicos: a) realizar diagnóstico e identificação de oportunidades de melhorias no fluxo de dados do SAM, por meio do Situation Analysis Canvas; b) propor adequações e melhorias ao fluxo de dados do SAM, a partir do emprego ferramenta Process Model Canvas e do alinhamento das características do Sistema ao modelo CDV-CI, c) mapear responsabilidades acerca da qualidade dos dados inseridos no SAM, a partir das melhorias propostas, por meio da Matriz $\mathrm{RACI}^{2}$.

\footnotetext{
${ }^{2}$ Acrônimo dos termos Responsible (Responsável), Accountable (Quem realiza a aprovação), Consulted (Quem deve ser consultado) e Informed (Quem deve ser informado) (WENDE; OTTO, 2007).
} 
Destaca-se que a escolha do modelo CVD-CI ter sido utilizado como base para esta pesquisa justifica-se:

a) pelo fato ter sido construído a partir do estudo e análise de uma série de modelos, entre eles: o modelo proposto pela Data Documentarion Initiative (DDI), o modelo proposto pela United Kingdon Data Archive (UKDA) e Economic and Social Data Service (ESDS), o modelo proposto pela Data Management Association (DAMA), e o modelo de ciclo de vida de curadoria de dados proposto pelo Digital Curation Centre (DCC);

b) por ter sido concebido objetivando atender as demandas da CI; e

c) pelo fato de estabelecer, além das fases do ciclo, o objetivo da qualidade de dados, um dos focos centrais da governança de dados e desta pesquisa.

Por meio desta pesquisa, almeja-se contribuir com o desenvolvimento da área da CI, sobretudo, acerca da temática CVD-CI e da governança aplicada à dados científicos, compartilhando as motivações de sua execução, a metodologia empregada e os resultados alcançados. Além disso, visa-se a influenciar outros estudos acerca da utilização de ferramentas de governança de dados no diagnóstico, na análise e na proposta de melhoria de problemas reais.

\section{CICLO DE VIDA DE DADOS}

Davenport (1998, p.19) define dados como "observações sobre o estado do mundo". São os elementos mais brutos (a matéria prima da informação), não tratados e sozinhos não possuem significado, como um número, uma palavra, ou uma imagem. Será um conjunto de dados, dotados de contexto, que irá gerar informação. Mas é preciso deixar claro que quando lhes faltar qualidade haverá um prejuízo para aqueles que utilizam a informação, principalmente para aqueles que dependem da informação para tomada de decisão.

Como exemplo podemos visualizar uma pesquisa eleitoral, na qual cada participante fornece ao pesquisador uma opinião (ou um conjunto de opiniões) sobre a eleição. O dado individualizado não fornece um resultado significativo, mas por meio do conjunto de vários entrevistados conseguimos extrair uma informação a respeito do possível resultado da eleição.

O dado é um importante insumo para gerar informações e conhecimentos (BARBIERI, 2011), tanto nas organizações como nos estudos científicos. Dessa forma, entende-se que deve ser uma preocupação constante dos gestores de sistemas viabilizar o acesso dos dados aos profissionais e aos pesquisadores interessados nestes, de forma a contribuir com a disseminação de informações e conhecimentos em ampla escala. 
Para garantir o acesso aos dados, Santana (2013) afirma que é necessário estudar e propor melhorias à todas as fases relacionadas ao acesso aos dados por meio de Tecnologias da Informação e Comunicação, desde o planejamento da coleta e/ou da criação dos dados, até a sua visualização pelos interessados.

Ainda na visão do autor, a CI é uma área que pode contribuir com a construção de novos arcabouços teóricos relacionados ao processo de acesso aos dados para atender à diferentes necessidades informacionais. Uma vez que a CI objetiva estudar a produção, armazenamento, disseminação e recepção da informação (dados com contexto), sobretudo com enfoque nas tecnologias empregadas durante esse processo (CAPURRO; HJORLAND, 2007).

Por meio de uma análise de diversos modelos de ciclo de vida de dados de desenvolvidos em estudos de áreas correlatas, Santana (2013) desenvolveu uma proposta de modelo de vida de dados que leva em consideração as características e especificidades da CI. Tal modelo foi denominado pelo autor de CVD-CI, e pode ser visualizado na Figura 1.

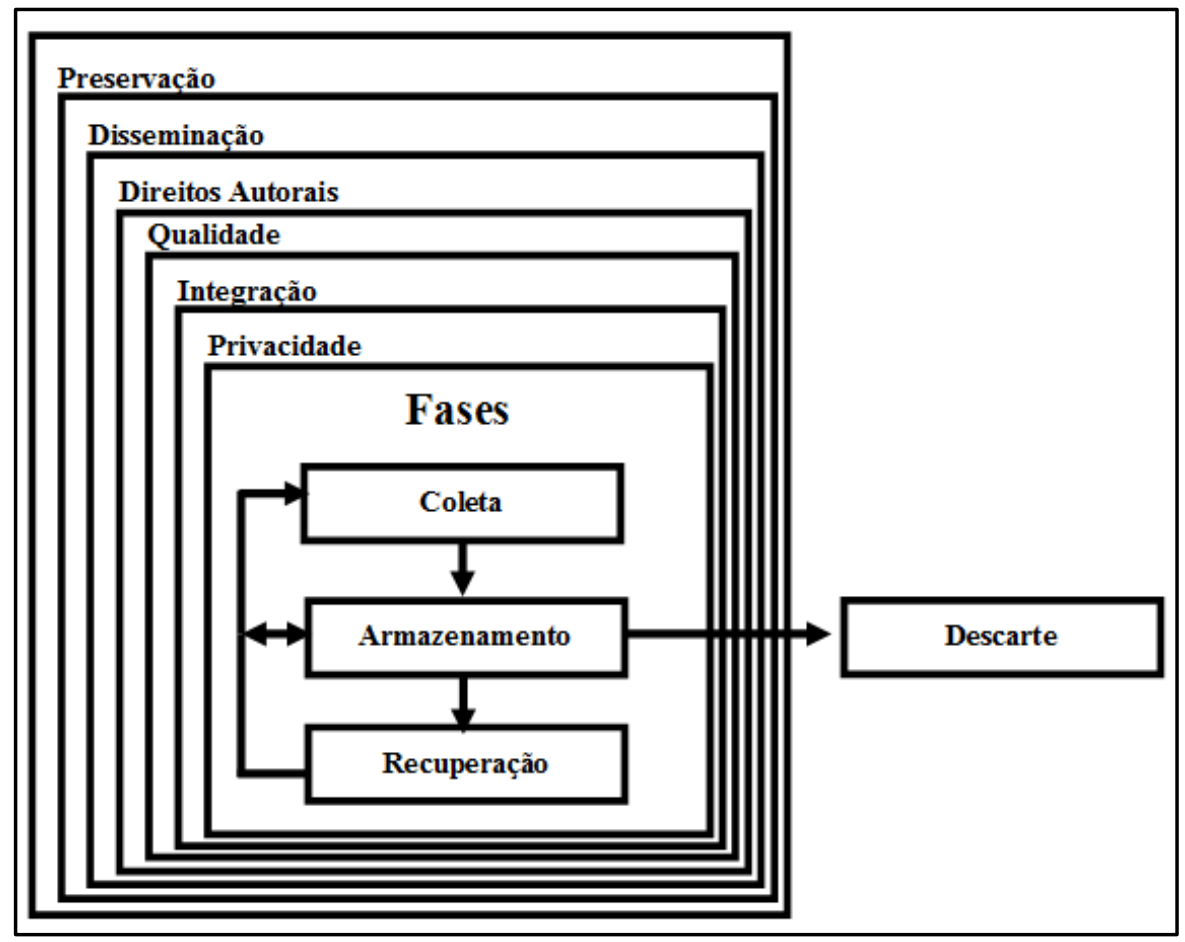

Figura 1. Diagrama representativo do CVD-CI Fonte: Santana (2013, p. 17)

O Modelo CVD-CI é composto por um processo de quatro fases: coleta, armazenamento, recuperação e descarte dos dados. As referidas etapas são permeadas por seis objetivos: privacidade, qualidade, direitos autorais, integração, disseminação e preservação. 
Salienta-se que as fases do mencionado Modelo serviram de embasamento para a identificação e a caracterização dos processos presentes no fluxo de dados do SAM, conforme será apresentado na metodologia deste artigo.

De acordo com o Modelo CVD-CI (SANTANA, 2013), na fase de coleta ocorre o planejamento inicial dos dados, bem como sua descrição por meio de metadados, sua avaliação e sua seleção. Na fase de armazenamento têm-se as atividades de processamento, transformação, inserção, modificação, transmissão, e demais ações relacionadas à preservação digital do dado. Já na recuperação há o acesso efetivo aos dados pelos profissionais e pesquisadores, ocorrendo assim as atividades de consulta e visualização dos dados, e ainda as ações de estruturação, filtro, tratamento, representação, refinamento e interatividade, as quais são realizadas após a obtenção dos dados. O descarte ocorre quando os objetivos previamente planejados para os dados são alcançados, ou ainda quando os limites de tempo de armazenamento previstos são atingidos.

O autor explica que "[...] a fase de coleta permite que seja iniciada a fase de armazenamento que por sua vez propicia a execução da fase de recuperação e ainda pode gerar novos dados retomando ações da fase de coleta." (SANTANA, 2013, p. 17). Dessa forma, a execução da fase de recuperação pode gerar dados que retroalimentam o ciclo, os referidos dados podem retomar ações da fase de coleta ou da fase de armazenamento.

\section{GOVERNANÇA DE DADOS}

A governança de dados pode ser definida como "o exercício da tomada de decisão e de autoridade para assuntos relacionados a dados" (DGI, c2017a, tradução nossa). Nesse sentido, a governança de dados determina políticas, acordos, papéis e responsabilidades com relação aos dados gerados na organização, bem como define quais métodos devem utilizados nas atividades de criação, armazenamento, avaliação, uso e eliminação de dados.

Conforme Loftis (2014) a governança de dados é uma estrutura que orienta e estabelece estratégias, políticas e objetivos com a finalidade de gerenciar os dados, como se fossem qualquer outro recurso de uma organização. Assim, para a governança de dados, os dados são importantes recursos para as organizações, os quais devem ser gerenciados, monitorados e acompanhados como qualquer outro insumo que auxilia no cumprimento da missão organizacional e no atingimento de seus objetivos.

Ainda de acordo com a autora, para implementar a governança de dados, deve-se conhecer a cultura organizacional e a forma como são tomadas as decisões na organização, para que assim o gestor consiga extrair vantagens da forma como sua empresa se comunica.

Dentre os objetivos da governança de dados, o DGI (c2017b) destaca sete: melhorar a tomada de decisão; reduzir conflitos operacionais; proteger as necessidades dos interessados

\begin{tabular}{l|l|l|l|l|l} 
(C) RDBCI: Rev. Digit. Bibliotecon. Cienc. Inf. & Campinas, SP & v.16 & n.3 & p. 274-298 & set./dez. 2018 \\
\hline
\end{tabular}


nos dados gerados; guiar os funcionários para adotar soluções comuns para as mesmas questões acerca de dados; construir padrões para processos; reduzir os custos e aumentar a eficácia por meio da coordenação dos esforços, e garantir a transparência dos processos.

A governança de dados possui também um importante papel de acompanhamento da gestão de dados na organização, como forma de garantir que os dados gerados nos processos estejam alinhados aos objetivos organizacionais. Além disso, monitora e acompanha os dados para que possam ser utilizados de forma eficaz e eficiente no atendimento dos objetivos organizacionais traçados pela alta administração.

Para cumprir esses objetivos, a governança de dados envolve um conjunto de processos, políticas, padronizações, organização e tecnologias necessárias para manipular e assegurar a disponibilidade, acessibilidade, qualidade, consistência, auditabilidade e segurança dos dados (SANTOS, 2010).

Dessa forma, ao adotar boas práticas de governança de dados, é possível planejar e implementar diretrizes, bem como padronizações internas que viabilizam a manipulação dos dados.

As boas práticas de governança de dados asseguram que os dados gerados nos processos institucionais estejam disponíveis a quem os necessitam, podendo ser acessados de forma rápida. A governança de dados visa também a garantir que os dados gerados sejam seguros, possuam consistência e qualidade, e possam ser auditados para diferentes finalidades.

Para Carvalho (2012) a prática da governança de dados, além de possibilitar o controle dos processos e dos métodos empregados na manipulação dos dados, permite prevenir situações adversas que possam comprometer a qualidade dos dados gerados na organização. Segundo a autora, por meio da governança de dados é possível aumentar a segurança, confidencialidade e qualidade dos dados, bem como a rapidez e a eficiência no seu tratamento.

Diante do exposto, acerca da governança de dados, entende-se que seus princípios e boas práticas podem ser aplicados também em processos que envolvam dados científicos, e que, portanto, não estejam diretamente relacionados à dados corporativos e ao alcance de objetivos organizacionais.

A governança aplicada à dados científicos está relacionada à planejar diretrizes, criar políticas, determinar papéis e responsabilidades, desenvolver estratégias e processos padronizados, visando que os dados possuam qualidade e estejam disponíveis para quem os necessita. 
Ao aplicar os princípios e as boas práticas da governança de dados no SAM, almeja-se que os dados inseridos, armazenados e disponibilizados pelo mencionado sistema de informação tenham mais qualidade e atendam mais eficazmente às necessidades dos PSE que o utilizaram.

A qualidade dos dados pode ser considerada um dos principais focos da governança de dados (BARBIERI, 2011). Diante da importância de disponibilizar dados confiáveis e de qualidade para os stakeholders de uma organização, bem como para os profissionais e os pesquisadores que utilizam dados científicos, será abordada a temática qualidade de dados.

\subsection{Qualidade de Dados}

Para auxiliar na compreensão do que é qualidade de dados, primeiramente recorremos à visão de Davok e Garcia (2014) acerca da qualidade. De acordo com as autoras, algo, como por exemplo um serviço, tem qualidade quando exibe valor e mérito. $\mathrm{O}$ valor está relacionado ao quanto o serviço é necessário para os stakeholders da organização, logo quanto mais os stakeholders necessitam do serviço, mais valor ele possui. O mérito está associado ao fato de utilizar recursos eficazmente e eficientemente para prestar o serviço, de maneira que atenda aos padrões de qualidade estabelecidos.

Assim, no contexto da qualidade dos dados em organizações, compreende-se que o dado tem qualidade, quando atende às necessidades dos stakeholders da instituição de onde ele é produzido, armazenado e utilizado. E também quando são empregados recursos (tecnológicos, pessoais e financeiros) de forma eficiente e eficaz para sua produção, gerenciamento e atendimento de padrões internos e externos de qualidade.

Wang e Strong (1996) afirmam que dados de qualidade são aqueles que estão aptos para o uso de seu consumidor. Mezzanzanica et al. (2015, p. 148) explicam que isso faz com que a qualidade dos dados seja um conceito que dependente do contexto em que está sendo abordada, pois "[...] um conjunto de dados pode ser considerado apropriado para um uso, embora possa não ser adequado para outro.”

Ao aplicar a definição de qualidade de Davok e Garcia (2014) a governança de dados, entende-se, por exemplo, que departamentos distintos que produzem dados idênticos, provocando a duplicação dos dados armazenados na organização, fazem com que esses dados não possuam mérito. Uma vez que tais dados são produzidos por meio de processos falhos e que provocam o desperdício de recursos da organização (ineficientes).

Ao duplicar os dados, faz-se com que a organização armazene dados que não são necessários para seus funcionários, gestores, acionistas, e demais grupos de interesse da organização, e que, portanto, não possuem valor. Logo, dados duplicados, além de não 
possuem mérito, também não apresentam valor, pois não atendem às necessidades dos stakeholders que os necessitam. Ou seja, dados duplicados não possuem qualidade.

Igualmente, entende-se que dados duplicados são prejudiciais para sistemas de informação que coletam, armazenam e recuperam dados científicos, uma vez que os dados duplicados não possuem valor para os pesquisadores e profissionais que vão utilizar o sistema. Assim como, dados duplicados em sistemas de informação não são meritórios, pois foram gerados por meio de processos ineficientes.

Além da duplicidade de dados, outros aspectos estão relacionados à baixa qualidade de dados como por exemplo: dados desatualizados, dados imprecisos, dados incompletos, entre outros.

Jesil̨evska (2017) apresenta algumas consequências da má qualidade de dados como: aumento dos custos operacionais empregados para detectar e corrigir erros ligados aos dados gerados, e baixa confiança nos dados, o que pode implicar em uma falta de aceitação dos usuários em iniciativas que utilizem tais dados.

Para avaliar a qualidade dos dados é necessária a determinação de atributos, os quais são denominados por Jesil̨evska (2017) como dimensões de qualidade de dados. Tais dimensões podem ser utilizadas para mensurar a qualidade de dados em diferentes contextos.

Dentre tais dimensões, têm-se, por exemplo: acurácia, temporalidade, consistência e completude dos dados. A acurácia diz respeito ao fato dos dados serem representativos da realidade (exatidão); a temporalidade está relacionada à atualidade dos dados e ao fato de estar acessível e disponível quando é necessário; a consistência faz referência à perseverança dos dados, ao fato destes não sofrerem mudanças, e a completude está associada aos dados estarem completos, sem valores nulos ou faltantes.

As dimensões da qualidade dos dados são importantes parâmetros para diagnosticar a situação atual relacionada aos dados e também para identificar o nível de qualidade dos dados que se almeja alcançar.

Diante disso, um dos enfoques desta pesquisa esteve relacionado a diagnosticar a qualidade dos dados disponibilizados no SAM, a partir de aspectos relacionados principalmente a completude, consistência e acurácia. Para isso, foram empregadas ferramentas para a governança de dados, as quais serão descritas a seguir. 


\subsection{Ferramentas para a Governança de Dados}

Compreende-se que um fator importante para auxiliar no controle da qualidade de dados é a determinação de papéis e de responsabilidades acerca dos dados. Dentre as ferramentas utilizadas para isto, têm-se a Matriz RACI, a qual apresenta os papéis envolvidos no processo e suas respectivas atribuições de responsabilidade.

Por meio dela, ocorre a documentação interna dos papéis e das responsabilidades dos membros das equipes, possibilitando pesquisas à ferramenta para sanar dúvidas e proporcionando diminuição dos conflitos entre as equipes (GRECO, 2014).

A Matriz RACI é utilizada por frameworks de governança como o Control Objectives for Information and related Technology (COBIT) e o Information Technology Infrastructure Library (ITIL) (FREITAS, 2013).

Ao empregar a Matriz RACI, proporciona-se benefícios como: contribuir para a divisão clara das tarefas entre os diferentes papéis envolvidos; evitar o esquecimento de pessoas-chave para o processo; melhorar a compreensão dos papéis e responsabilidades associadas ao processo; servir de comunicação interna, e sistematizar em um documento formal as responsabilidades dos envolvidos no processo (SMITH; ERWIN, [2005]; RIVERO NETO, [201-?]; GRECO, 2014).

Dessa forma, a Matriz RACI é uma importante ferramenta que pode ser aplicada na governança de dados, auxiliando na determinação de papéis e de responsabilidades dos envolvidos nas atividades, nos processos e nos setores das organizações.

Outra ferramenta que pode ser empregada na governança de dados é o Situation Analysis Canvas, em português Quadro de Análise Situacional. A mencionada ferramenta pode ser aplicada de forma individual ou em conjunto com outras ferramentas, como por exemplo a Análise SWOT (COLOMBI, 2015).

Ainda conforme o autor, por meio do Situation Analysis Canvas é possível traçar um panorama da situação a ser estudada, apresentando um diagnóstico atual e elencando as ações prioritárias (que devem ser realizadas para alcançar o cenário desejado) e os resultados almejados.

Para isso, o Situation Analysis Canvas apresenta um quadro composto por sete componentes responsáveis por auxiliar no diagnóstico e na análise da situação a ser estudada: situation analysis (análise situacional); challenges and consequences (desafios $\mathrm{e}$ consequências); pivotal questions (questões fundamentais); recommended approach (abordagem recomendada); reasons to believe (razões para acreditar); how to make it happen (como fazer isso acontecer), e next steps (próximos passos) (COLOMBI, 2015).

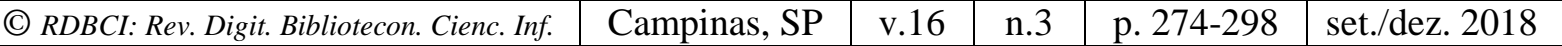


Entende-se que o Situation Analysis Canvas possibilita para a governança de dados um diagnóstico completo da situação estudada, proporcionando a análise dos desafios e de suas consequências, e auxiliando no planejamento de quais ações devem ser adotadas para melhorar o fluxo de dados atual, visando a garantir que os dados estejam disponíveis com qualidade para todos os interessados.

Uma terceira ferramenta que pode ser empregada na governança de dados é o Process Model Canvas, em português Quadro de Modelo de Processo, o qual foi desenvolvido por Marco Bijl, James P. Devlin e David Ruting (PROCESS..., c2014).

O Process Model Canvas foi criado como complemento do Business Model Canvas, em português Quadro de Modelo de Negócios, modelo desenvolvido por Alexander Osterwalder como uma ferramenta de gerenciamento estratégico. O Business Model Canvas proporciona um esquema visual que pode ser empregado para compreender o funcionamento de um negócio já existente, ou para o planejamento de um negócio a ser desenvolvido (MOURA, 2014).

Ao aplicar o Process Model Canvas em governança de dados é possível sistematizar em um único documento: as razões que motivam alguém a utilizar os dados; a proposta de valor atribuída aos dados; o que é necessário fazer para o valor desejado seja entregue ao interessado nos dados; qual o fluxo de informação necessário para isso acontecer; qual o valor recebido ao final do processo pelo interessado nos dados, e qual a sua reação ao receber esse valor (PROCESS... c2014).

Assim, a mencionada ferramenta possibilita conectar o gerenciamento estratégico ao gerenciamento operacional (ibid), proporcionando alinhar o que é planejado pela alta administração com o que deve ser executado para que os interessados nos dados fiquem satisfeitos ao final do processo.

Destaca-se que nesta subseção foram apresentados somente algumas das ferramentas existentes que podem ser aplicadas na governança de dados. O enfoque esteve relacionado às ferramentas empregadas neste estudo, sendo a maneira como foram aplicadas pelos seus autores descrita a seguir.

\section{METODOLOGIA}

Quanto aos aspectos metodológicos, entende-se que esta pesquisa pode ser caracterizada como aplicada, com caráter exploratório, coleta de dados por meio de pesquisaação, e análise dos dados por meio de métodos qualitativos. 
De acordo com Silva e Menezes (2005) a pesquisa aplicada tem por finalidade gerar conhecimentos para aplicação prática e para solução de problemas específicos. Dessa forma, essa pesquisa é aplicada, pois busca a solução de um problema prático, relacionado ao diagnóstico, análise e proposta de melhoria para o SAM, com vista a proporcionar mais qualidade aos dados submetidos, armazenados e disponibilizados no Sistema.

Quanto aos objetivos, esta pesquisa pode ser classificada como exploratória, pois visa a proporcionar maior familiaridade com o objeto de estudo, buscando torná-lo mais explícito (VIEIRA, 2002). Por meio desta pesquisa, almejou-se reunir informações acerca dos processos de submissão, armazenamento e visualização dos dados de avaliação motora do SAM, como forma de explorar os problemas enfrentados nesses processos e propor melhorias, com vista a proporcionar mais qualidade aos dados disponibilizados no Sistema.

Para o alcance dos objetivos específicos propostos, empregou-se a abordagem metodológica pesquisa-ação, a qual é caracterizada por Lima (2007) como a busca por uma solução inovadora de um problema real, mas que ao contrário do Estudo de Caso, requer envolvimento ativo do pesquisador e dos pesquisados no contexto do objeto de estudo.

Conforme o autor, a pesquisa-ação possui base empírica, e é voltada para a aplicação prática, sendo composta por um processo cíclico, denominado de investigação-ação, que consiste basicamente em: constatar um problema; raciocinar sobre uma solução para esse; planejar sua execução; executá-la e avaliar os resultados encontrados para retroalimentar o sistema, visando a melhoria do processo.

O princípio do ciclo investigação-ação, descrito por Lima (2007), pode ser observado também no estudo de Lorences e Ávila (2013), em que as autoras propõem um procedimento para avaliação e para melhoria da governança de Tecnologia da Informação (TI) em organizações. Tal procedimento foi desenvolvido com foco na governança de TI, no entanto, compreende-se que pode ser aplicado igualmente na governança de dados, uma vez que esta constitui-se de uma extensão dos conceitos de governança corporativa e da governança de TI (BARBIERI, 2011).

O procedimento de Lorences e Ávila (2013) é constituído por quatro fases: avaliação, design, implementação e controle. A fase de avaliação é responsável, entre outras atividades, pelo diagnóstico atual da governança na organização, pela caracterização da organização e pela análise do alinhamento dos recursos de TI aos objetivos da organização. Na fase de design é realizada a modelagem e a análise dos processos de TI, permitindo a identificação de oportunidades de melhoria nos processos, a partir dos resultados da fase anterior. Na fase de implementação ocorre a execução do processo modelado, e na fase de controle há a avaliação do processo implementado com vistas a melhorá-lo continuamente. 
Tendo em vista o procedimento proposto pelas autoras, bem como as etapas de uma pesquisa-ação descritas por Lima (2007), primeiramente, foi realizado o diagnóstico situacional do SAM, por meio de informações levantadas pela vivência de um dos autores deste estudo, o qual foi responsável pelo desenvolvimento do Sistema e por sua disponibilização on-line. Para esta primeira fase, utilizou-se o diagrama Situation Analysis Canvas $^{3}$.

Por meio do Situation Analysis Canvas, foi possível realizar uma avaliação das dificuldades acerca do SAM, traçando os desafios a serem enfrentados e as consequências desses desafios, determinando os problemas centrais a serem resolvidos no Sistema, o que fazer para solucioná-los, bem como os próximos passos que devem ser seguidos para isso acontecer.

Na segunda fase, identificou-se, por meio do Situation Analysis Canvas e do CVD-CI (SANTANA, 2013), três processos do SAM que poderiam ser melhorados para contribuírem, de forma mais efetiva, com o seu objetivo de disseminar dados de qualidade relacionados à avaliação motora de crianças e idosos.

Os processos identificados foram: submissão, armazenamento e visualização dos dados. Optou-se, no entanto, por adaptar a nomenclatura da etapa de coleta descrita no CVDCI para submissão, e da etapa recuperação para visualização dos dados, por entender que tais nomenclaturas são mais adequadas ao contexto do SAM.

Além disso, a etapa do CVD-CI de descarte dos dados não foi incluída nesta pesquisa, pois entende-se que é finalidade do SAM ter uma série histórica dos dados para auxiliar no diagnóstico e acompanhamento do desenvolvimento motor dos pacientes atendidos pelos profissionais que utilizam o Sistema.

$\mathrm{Na}$ terceira fase, desenvolveu-se um estudo acerca de cada um dos processos identificados, visando a compreender melhor suas deficiências e quais ações poderiam ser traçadas para sanar tais problemas.

Para isso empregou-se o Process Model Canvas, ele foi determinado para cada processo do SAM identificando: as razões que motivam os PSE a usarem tal processo; o valor que se deseja entregar para esses profissionais; o que é necessário fazer para que o valor desejado seja alcançado; qual o fluxo de informação necessário para os mencionados profissionais receberem o valor desejado; qual o valor entregue a eles no final do processo, e qual a reação desses profissionais ao receber os dados de avaliação motora no final do processo.

\footnotetext{
3 Destaca-se que os diagramas empregados neste estudo e apresentados na seção dos resultados foram desenvolvidos por meio da ferramenta de edição on-line Piktochart, a qual está disponível em: https://piktochart.com/.

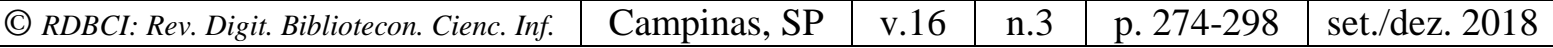


Por fim, criou-se uma proposta de Matriz RACI sobre as responsabilidades da qualidade dos dados inseridos no SAM, a partir de diferentes papéis como: os PSE, e o Centro de Tecnologia da Informação (CTI) responsável pela gestão do Sistema.

Para obtenção dos resultados, empregaram-se procedimentos qualitativos de análise dos dados, os quais de acordo com Martins e Theóphilo (2009) incluem a descrição, compreensão e interpretação dos dados. O emprego de procedimentos qualitativos, deu-se pelo fato dos dados coletados serem predominantemente descritivos e requerem análise que não pode ser expressa em números.

\section{RESULTADOS}

O SAM foi inicialmente desenvolvido, e ainda se encontra em processo de construção, com dois grandes objetivos:

a) coletar dados gerados a partir dos testes de avaliação motora, realizados utilizando protocolo EDM, de forma informacional;

b) expor as informações coletadas, de forma coletiva, à sociedade como um todo (poder público, comunidade acadêmico/científica e setor privado).

Alcançar ambos os objetivos têm por finalidade trazer eficiência, segurança e confiabilidade (por meio da informatização) na realização dos testes, e apresentar à sociedade uma ferramenta de análise que permitirá o direcionamento de políticas públicas, dados para realização de novas pesquisas científicas, além de métricas de medição do avanço da saúde do paciente.

Para compreender o funcionamento do SAM, sobretudo o fluxo de seus dados, primeiramente, realizou-se uma análise com enfoque na situação atual dos cadastros dos PSE que o utilizam, nos mecanismos empregados para avaliar a qualidade dos dados inseridos, e na disponibilização dos dados. O diagrama resultante dessa análise pode ser observado na Figura 2. 


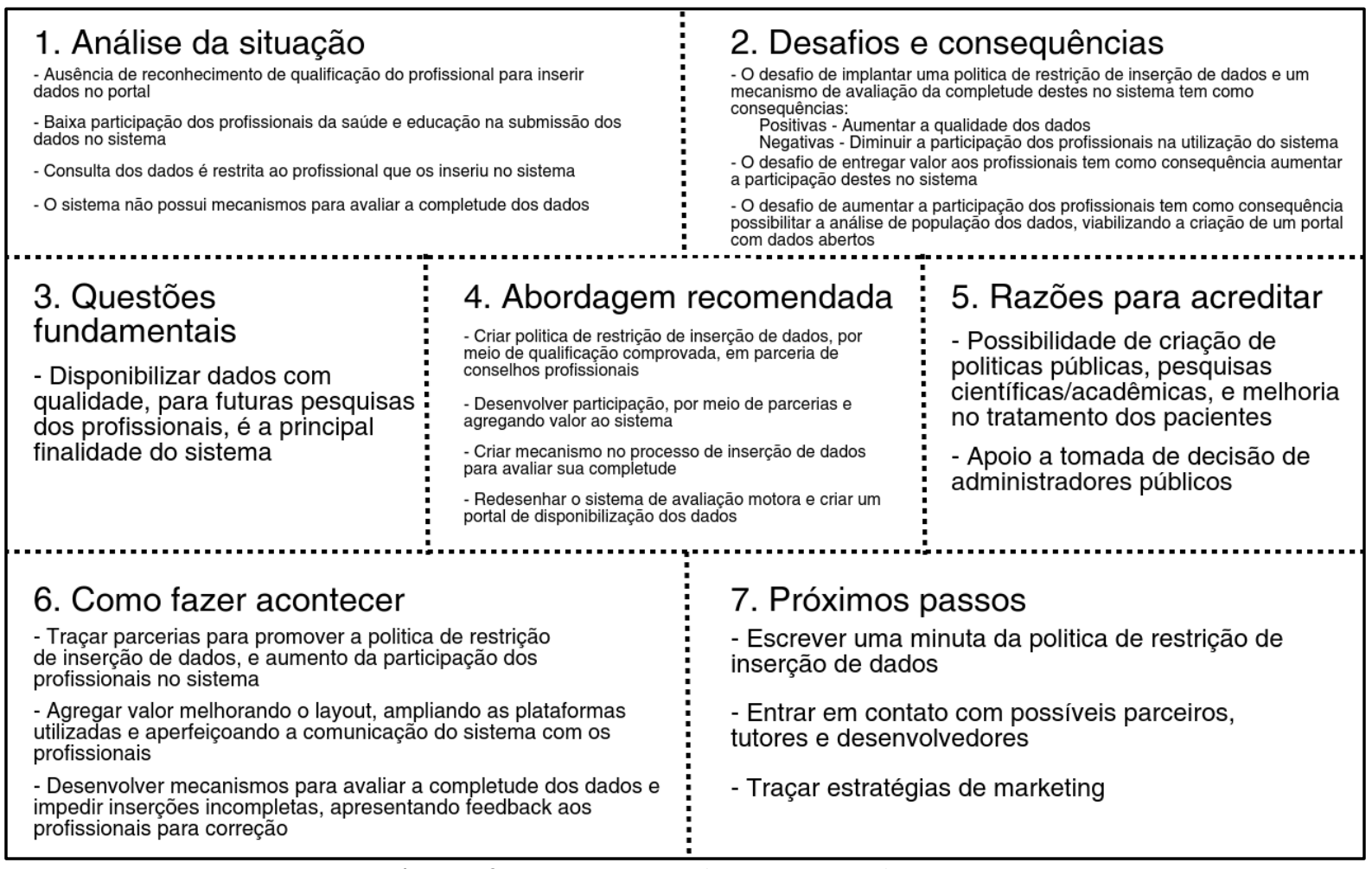

Figura 2. Situation Analysis Canvas do SAM Fonte: Dos autores (2018)

A partir da análise situacional realizada por meio do Situation Analysis Canvas, presente na Figura 2, foi possível verificar que atualmente para utilizar o SAM é necessário solicitar cadastro para os moderadores do Sistema. Após a análise dos dados pelos moderadores, o profissional tem sua solicitação de acesso negada ou autorizada. No entanto, o atual processo de cadastro para utilizar o SAM não leva em consideração a aptidão destes para inserir os dados. De forma que o profissional pode acabar por inserir dados com baixa qualidade, por ignorar alguma particularidade do Sistema ou do método de avaliação motora EDM.

Diante disso, detectou-se a necessidade de criar e implementar uma Política de inserção de dados, restringindo a inclusão de dados somente para profissionais qualificados, por meio da validação cruzada dos Registros Profissionais em parceria de Conselhos Profissionais da área da saúde e da educação. A comprovação de aptidão para inserir dados e utilizar o SAM também será por meio da aprovação no curso on-line à distância de “Avaliação do Desenvolvimento Motor Infantil" oferecido pela Sociedade Brasileira de Motricidade Humana (SBMH) ${ }^{4}$.

Ainda por meio do Situation Analysis Canvas, observou-se a baixa participação dos PSE na utilização do SAM. Uma vez que o Sistema não é a única ferramenta disponível para o registro de dados de avaliação motora. Dessa forma, identificou-se a necessidade de realizar

${ }^{4}$ Para mais informações: https://ead.motricidade.com.br/curso/desenvolvimento-infantil

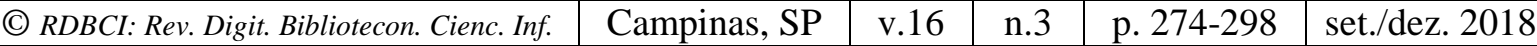


campanhas de divulgação do SAM entre o seu potencial público alvo, bem como buscar parcerias com órgãos de classe. Além disso, constatou-se ser indispensável agregar valor à utilização do Sistema, de forma que os PSE se sintam motivados a utilizá-lo, ao invés das outras ferramentas disponíveis.

Verificou-se ser indispensável redesenhar o SAM, com vistas a criar um portal de acesso aberto aos dados de avaliação motora de crianças e idosos, uma vez que, atualmente, somente os profissionais cadastrados no sistema podem visualizar os dados. Cada profissional possui acesso somente aos dados inseridos por ele mesmo, o que impossibilita que profissionais interessados em análises estatísticas para o desenvolvimento de pesquisas e estudos científicos tenham acesso a totalidade de dados registrados no Sistema.

Por fim, apurou-se a ausência de mecanismos de avaliação da completude dos dados inseridos no SAM. Consequentemente, aumenta-se a chance de inserção de dados com valores nulos ou em branco no Sistema, fator que pode afetar a qualidade dos dados armazenados. Por isso, compreende-se que ao redesenhar o SAM, deve-se planejar, desenvolver e implementar ferramentas adequadas para verificação e controle da completude dos dados.

Na sequência, ao avaliar o fluxo completo dos dados, foram identificados três grandes processos, distintos entre si (no valor proposto, público alvo e funcionamento da plataforma). Portanto foram gerados três Process Model Canvas: Submissão dos dados (Figura 3); armazenamento dos dados (Figura 4); visualização dos dados (Figura 5).

\section{PROCESSO DE SUBMISSÃO DOS DADOS}

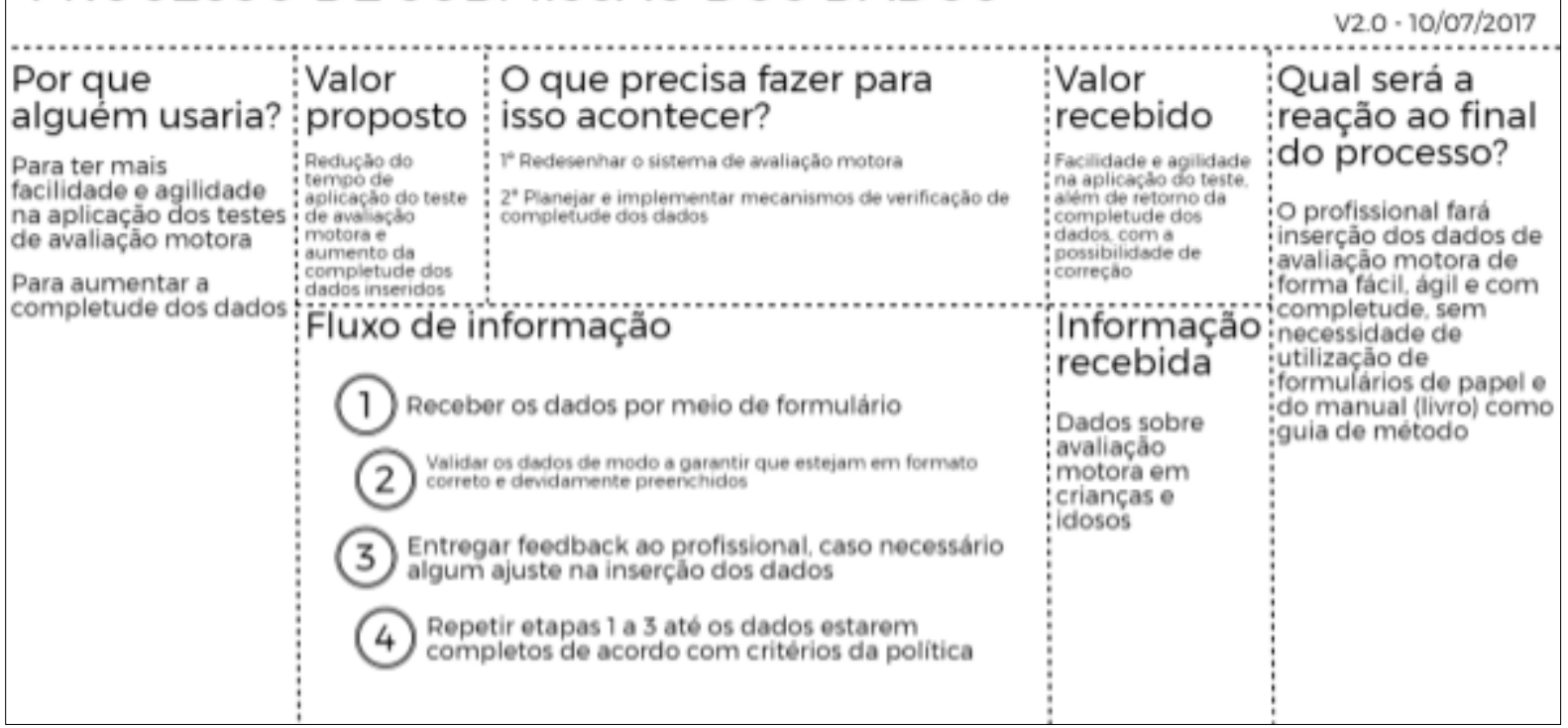

Figura 3. Process Model Canvas de submissão dos dados

Fonte: Dos autores (2018) 
Por meio do Process Model Canvas de submissão dos dados (Figura 3), entende-se que o valor proposto do SAM está relacionado a promover facilidade e agilidade na aplicação dos testes de avaliação motora aos PSE que buscam inserir dados, além de aumentar a completude dos dados inseridos, reduzindo o tempo de aplicação dos testes. Para tal será preciso redesenhar o sistema e planejar/implementar mecanismos de verificação de completude dos dados.

A Figura 3 também auxiliou na visualização do fluxo de informação por qual os dados passarão após o redesenho do SAM, sendo: receber os dados por meio de formulário preenchido pelo profissional; validar os dados, por meio do sistema informatizado, para que estejam em formato correto e devidamente preenchido; apresentar feedback, ainda por meio do sistema informatizado, sobre os dados inseridos; repetir as etapas 1 a 3 até os dados estarem devidamente ajustados de acordo com critérios pré-definidos.

Na Figura 4, Process Model Canvas de armazenamento dos dados, o valor apresentado ao profissional para utilizar este serviço recai na confiança de que seus dados estarão armazenados de forma segura, por meio digital, e que passarão por tratamento que evitará duplicidade e garantirá homogeneidade. Compreende-se que, para que isto aconteça, o SAM precisa ser alterado, isto é, faz-se necessário o redesenho do seu funcionamento, desenvolvendo e implantando o sistema de armazenamento e tratamento dos dados.

\section{PROCESSO DE ARMAZENAMENTO DOS DADOS}

\begin{tabular}{|c|c|c|c|c|}
\hline & $\begin{array}{l}\text { Valor } \\
\text { proposto } \\
\text { propos } \\
\text { Armazenar os } \\
\text { dados com } \\
\text { segurança, } \\
\text { confiança de que } \\
\text { poderáoser } \\
\text { recuperados e } \\
\text { tratados } \\
\text { reducindo erros }\end{array}$ & & & \\
\hline $\begin{array}{l}\text { Por que } \\
\text { alguém usaria? } \\
\text { Para ter os dados } \\
\text { armazenados em meio } \\
\text { digital } \\
\text { Tratamento dos dados } \\
\text { para evitar duplicidade } \\
\text { e garantir } \\
\text { homogeneidade } \\
\text { Processamento dos } \\
\text { dados para } \\
\text { preservação digital }\end{array}$ & \multicolumn{2}{|c|}{$\begin{array}{l}\text { (1) Armazenar os dados em um banco } \\
\text { (2) Tratar os dados armazenados de modo } \\
\text { outrosogeneizá-los, evitar duplicidade e }\end{array}$} & $\begin{array}{l}\text { Informação } \\
\text { recebida } \\
\text { Dados tratados, } \\
\text { homogenizados, } \\
\text { com redução de } \\
\text { erros e } \\
\text { disponiveis para } \\
\text { consulta }\end{array}$ & $\begin{array}{l}\text { Qual será a } \\
\text { reação ao final } \\
\text { do processo? } \\
\text { o profissional terá } \\
\text { confiança de que seus } \\
\text { dados de avaliação } \\
\text { motora estarão } \\
\text { armazenados em um } \\
\text { banco de dados } \\
\text { digital, que passaram } \\
\text { por um tratamento } \\
\text { visando a } \\
\text { homogeneidade e } \\
\text { ievitando a } \\
\text { duplicidade. Bem } \\
\text { como tratados para } \\
\text { garantir a preservação } \\
\text { digital }\end{array}$ \\
\hline
\end{tabular}

Figura 4. Process Model Canvas de armazenamento dos dados Fonte: Dos autores (2018) 
Como fluxo de informação foi possível visualizar, inicialmente, o armazenamento dos dados em um banco de dados, para posteriormente serem tratados por meio do sistema informatizado que irá homogeneizá-los, evitando erros e eliminando duplicidades.

Por fim, no último processo mapeado (Figura 5) referente a visualização dos dados, entende-se que o valor entregue aos PSE que utilizam o SAM está relacionado a visualização de dados confiáveis sobre avaliação motora. Tais dados auxiliariam na realização de pesquisas científicas na área, bem como apoiariam no planejamento de ações/políticas públicas que promovam o tratamento de distúrbios do desenvolvimento humano, além de impactar no processo de tomada de decisão sobre assuntos relacionados à motricidade da população.

\section{PROCESSO DE VISUALIZAÇÃO DOS DADOS}

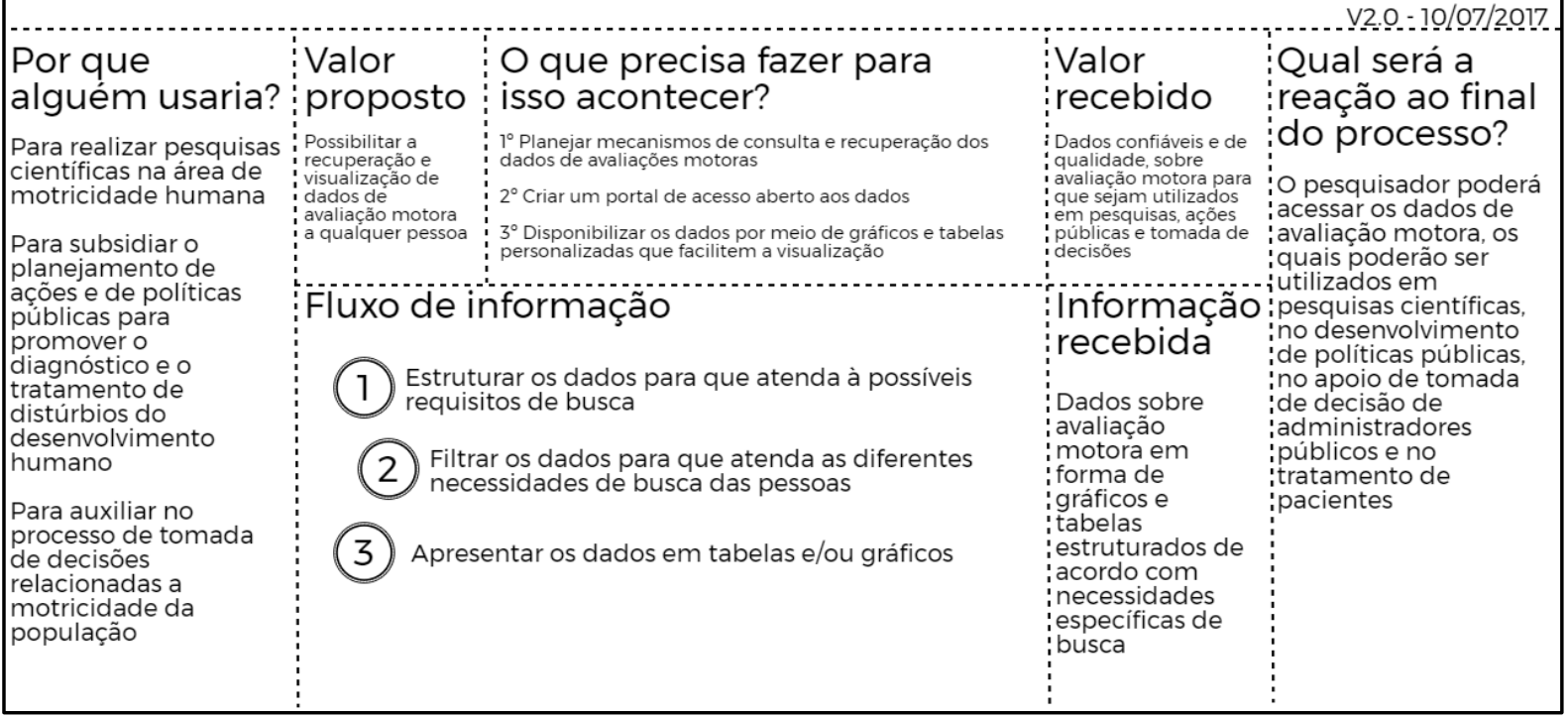

Figura 5. Process Model Canvas de visualização dos dados Fonte: Dos autores (2018)

O fluxo de informação apresentado pela Figura 5 inicia na estruturação dos dados, seguido da filtragem com base nas necessidades do profissional, e por fim na apresentação destes dados em formato de tabela e/ou gráficos.

A Figura 6 contém a proposta da Matriz RACI desenvolvida para o SAM.

\begin{tabular}{|c|c|c|c|c|c|}
\hline (C) RDBCI: Rev. Digit. Bibliotecon. Cienc. Inf. & Campinas, SP & v.16 & n.3 & p. $274-298$ & \\
\hline
\end{tabular}




\begin{tabular}{|c|c|c|c|c|}
\hline \multicolumn{5}{|c|}{ MATRIZ DE RESPONSABILIDADE } \\
\hline MATRIZ RACI & 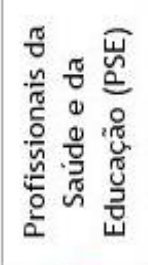 & 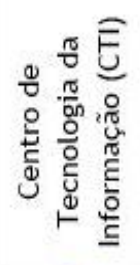 & 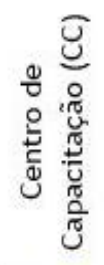 & 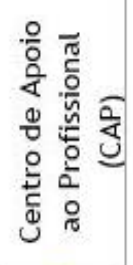 \\
\hline Qualidade dos dados nas fontes de informação. & & $\mathbf{s}$ & $\mathbf{P}$ & $\mathbf{P}$ \\
\hline Completude & $\mathbf{P}$ & $y$ & $\mathbf{P}$ & $\mathbf{P}$ \\
\hline Consistência & & $\mathbf{s}$ & $\mathbf{P}$ & $\mathbf{P}$ \\
\hline Acurácia & & $\mathbf{s}$ & $\mathbf{P}$ & $\mathbf{P}$ \\
\hline
\end{tabular}

Figura 6. Proposta de Matriz RACI para o SAM

Fonte: Dos autores (2018)

Legenda: $\mathrm{R}=$ Papel responsável pela execução/controle; $\mathrm{A}=$ Papel responsável por aprovar; $\mathrm{S}=$ Papel responsável por supervisionar; $\mathrm{P}=$ Papel responsável por participar, caso for necessário.

Na criação da matriz RACI focamos na avaliação da distribuição de responsabilidades referentes a qualidade dos dados, subdividindo este item em três que, posteriormente, influenciaram no resultado do principal. Para avaliar cada subitem identificamos os profissionais envolvidos com a qualidade dos dados, sendo: os PSE, que inserem os dados no SAM; o CTI, criador do SAM; o Centro de Capacitação (CC), que treina aqueles que irão inserir dados no SAM; o Centro de Apoio ao Profissional (CAP), responsável por prestar suporte aos profissionais que utilizam o SAM.

Completude foi o primeiro subitem avaliado. Foi definido o CTI como responsável por este item pois ele deve impedir, por meio da tecnologia utilizada no SAM, que qualquer dado incompleto seja inserido no sistema. Enquanto os outros três envolvidos são apenas participantes.

Ao avaliar consistência e acurácia entendemos que o responsável por ambos é o PSE. Ele é o único que tem acesso à fonte do dado, portanto o único que tem a capacidade de inserir estes dados de forma coerente e o mais próximo do valor real. Qualificamos o CTI como supervisor em ambos os itens, para que acompanhe possíveis sinais de desvios de consistência e acurácia dos dados, e o CC e CAP como participantes.

Por fim a distribuição de responsabilidade sobre a qualidade dos dados foi realizada com base nos itens que mais apareceram para cada envolvido nos subitens completude, consistência e acurácia. 


\section{CONSIDERAÇÕES FINAIS}

Ao fim deste artigo, ressalta-se que os objetivos propostos foram realizados, uma vez que foi possível diagnosticar e identificar oportunidades de melhoria e propor adequações ao fluxo de dados do SAM, além de mapear responsabilidades acerca da qualidade dos dados.

Por meio da governança de dados, com o emprego de ferramentas como o Situation Analysis Canvas e o Process Model Canvas, além da Matriz RACI, foi possível evidenciar falhas no formato atual de segurança dos dados do SAM, responsabilidades alocadas de forma errônea entre os envolvidos, e principalmente erros no conceito, planejamento e desenvolvimento da ferramenta.

Tais problemas que, agora visíveis, poderão ser sanados ao passo que os responsáveis pelo SAM terão subsídios para executar uma nova versão da plataforma, que terá como objetivo agregar valor:

a) facilitando a inserção de dados das avaliações motoras para os profissionais, proporcionando mais agilidade ao processo e maior completude aos dados armazenados;

b) auxiliando o profissional na eliminação de dados duplicados, além de proporcionar ferramentas que garantem a homogeneidade destes;

c) garantindo a segurança e preservação digital das informações inseridas, pelo profissional, no sistema;

d) permitindo uma pesquisa intuitiva de dados, para os profissionais envolvidos na inserção do Sistema e para a sociedade como um todo, auxiliando-os no desenvolvimento de pesquisas científicas, políticas públicas, e melhor tratamento dos pacientes.

Portanto, conclui-se por meio desta pesquisa, a importância e o valor que se agrega à um sistema de informação quando se trabalha com ferramentas específicas de governança de dados, permitindo a quem as utilizam a criação de sistemas mais eficientes, e/ou então auxiliando na evolução de sistemas existentes que não tiveram a oportunidade de empregar estas ferramentas durante sua criação, como é o caso do SAM.

Acredita-se que ao apresentar à comunidade científica esta pesquisa ação, com exemplo prático, estamos contribuindo para a formação do conhecimento da CI.

Igualmente, compreende-se que, por meio deste trabalho, poderá criar subsídios e até mesmo incentivar a realização de outras pesquisas acerca da busca de soluções para problemas reais, que possam aplicar os princípios e as ferramentas da governança de dados, bem como os conceitos do CVD-CI. 
Por fim, deixa-se como sugestão de estudo futuro a análise, por meio de um estudo de caso, do SAM, com o intuito de coletar novos dados, e de avaliar como está a performance da nova plataforma, em relação ao sistema antigo. Além de verificar quais foram os benefícios obtidos com as mudanças realizadas, potenciais problemas gerados e novas análises com sugestões de melhoria.

\section{REFERÊNCIAS}

BARATA, André Montoia. Governança de dados em organizações brasileiras: uma avaliação comparativa entre os benefícios previstos na literatura e os obtidos pelas organizações. 2015. Dissertação (Mestrado em Ciências) - Programa de Pós-Graduação em Sistemas de Informação, Escola de Artes, Ciências e Humanidades, Universidade de São Paulo, São Paulo, 2015. 155 f. Disponível em: <http://www.teses.usp.br/teses/disponiveis/100/100131/tde-28072015-215618/pt-br.php>. Acesso em: 06 ago. 2017.

BARBIERI, Carlos. BI2: Business Intelligence: modelagem e qualidade. Rio de Janeiro: Elsevier, 2011.

CAPURRO, Rafael; HJORLAND, Birger. O conceito de informação. Perspec. Ci. Inf., Belo Horizonte, v. 12, n. 1, p. 148-207, jan./abr. 2007. Disponível em: $<$ http://portaldeperiodicos.eci.ufmg.br/index.php/pci/article/view/54/47>. Acesso em: 08 ago. 2017.

CARVALHO, Mónica Isabel Machado. Data Governance: estudo e aplicação na EDP distribuição. 2012. Relatório de Estágio (Mestrado em Gestão) - Faculdade de Economia, Universidade de Évora, Évora, 2012. 57 p. Disponível em:

<http://hdl.handle.net/10316/21346>. Acesso em: 20 jul 2017.

COLOMBI, Luca. Marketing performance measurement: how to build a situation analysis. London: Conduit Data Services, 2015. Disponível em: $<$ http://www.conduithub.com/marketing-performance-measurement-using-the-situationanalysis-canvas >. Acesso em: 09 set. 2017.

CORRÊA, Fabiano Couto. O papel dos bibliotecários na gestão de dados científicos. R. Digit. Bibliotecon. Ci. Inf., Campinas, v.14, n.3, p.387-406, set/dez. 2016. Disponível em: <http://dx.doi.org/10.20396/rdbci.v14i3.8646333 >. Acesso em: 29 jul. 2017.

DAVENPORT, Thomas. H. Ecologia da informação: por que só a tecnologia não basta para o sucesso na era da informação. São Paulo: Futura, 1998.

DAVOK, Delsi Fries; GARCIA, Renata Ivone. Modelo de avaliação de valor e mérito de estoques de informação de bibliotecas universitárias. Enc. Bibli: R. Eletr. Bibliotecon. Ci. Inf., Florianópolis, v. 19, n. 39, p. 19-42, jan./abr., 2014. Disponível em: <http://dx.doi.org/10.5007/1518-2924.2014v19n39p19>. Acesso em: 04 ago. 2017. 
DATA GOVERNANCE INSTITUTE (DGI). Definitions of Data Governance. [S.1.]: DGI, c2017a. Disponível em:

<http://www.datagovernance.com/adg_data_governance_definition/>. Acesso em: 20 jul.

2017.

DATA GOVERNANCE INSTITUTE (DGI). Goals and Principles for Data Governance. [S.1.]: DGI, c2017b. Disponível em:

<http://www.datagovernance.com/adg_data_governance_goals/>. Acesso em: 20 jul. 2017.

FREITAS, Marcos André dos Santos. Fundamentos do gerenciamento de serviços de TI. 2. ed. Rio de Janeiro: Brasport, 2013.

GRECO, Rafael Escaleão. Governança de TI aplicada em Prefeitura: modelagem e melhoria dos processos de negócio utilizando boas práticas do Cobit. 2014. 66 p. Monografia (Bacharelado em Sistemas de Informação) - Fundação de Ensino Eurípides Soares da Rocha, Centro Universitário Eurípides de Marília. Marília: UNIVEM, 2014. Disponível em: <http://hdl.handle.net/11077/1010 . Acesso em: 08 set. 2017.

JESILEVSKA, Svetlana. Data Quality Dimensions to Ensure Optimal Data Quality. The Romanian Economic Journal. v. 20, n. 63, mar. 2017. p. 89-103. Disponível em: $<$ http://www.rejournal.eu/sites/rejournal.versatech.ro/files/articole/2017-0402/3443/6jesilevska.pdf>. Acesso em: 13 set. 2017.

LIMA, João Alberto de Oliveira. Pesquisa-ação em Ciência da Informação. In: MUELLER, Suzana Pinheiro Machado (Org.). Métodos para a pesquisa em Ciência da Informação. Brasília: Thesaurus, 2007. p. 63-82. (Série Ciência da Informação e da Comunicação).

LOFTIS, Lisa. O que é governança de dados? [S.1.]: Youtube, 2014. Disponível em: <https://www.youtube.com/watch?v=icIgspqFbFo\&t=18s $>$. Acesso em: 20 jul. 2017.

LORENCES, Patricia Pérez; ÁVILA, Lourdes Francisca García. The evaluation and improvement of it governance. JISTEM: J. Inf. Syst. Technol. Manag., São Paulo, v. 10, n. 2, p. 219-234, ago. 2013. Disponível em: <http://dx.doi.org/10.4301/S180717752013000200002>. Acesso em: 07 set. 2017.

MARTINS, Gilberto de Andrade; THEÓPHILO, Carlos Renato. Metodologia da investigação científica para ciências sociais aplicadas. 2. ed. São Paulo: Atlas, 2009.

MEZZANZANICA, Mario. et al. A model-based evaluation of data quality activities in KDD. Information Processing \& Management, v. 51, n. 2, mar. 2015, p. 144-166. Disponível em: 〈http://dx.doi.org/10.1016/j.ipm.2014.07.007〉. Acesso em: 06 ago. 2017.

MOURA, Luiz Antonio Rolim de. Memória Seminário sobre Canvas Model com Alexander Osterwlader. [S.1.]: SEBRAE Paraná, 2014. Disponível em: $<$ https://pt.slideshare.net/luizrolimdemoura/memoria-seminario-sobre-canvas-model-comalexander-osterwlader-by-luiz-rolim>. Acesso em: 09 set. 2017. 
PROCESS Model Canvas. [S.1.: s.n], c2014. Disponível em:

<http://www.processmodelcanvas.com/>. Acesso em: 09 set. 2017.

RIVERO NETO, Miguel. Matriz RACI: entenda o que é e como usar. Palhoça: VEC, [201?]. Disponível em: <http://valorecompetencia.com.br/gestao-de-operacoes/matriz-racientenda-o-que-e-e-como-usar>. Acesso em: 06 ago. 2017.

SANTANA, Ricardo Cesar Gonçalves. Ciclo de vida dos dados e o papel da ciência da informação. In: ENCONTRO NACIONAL DE PESQUISA EM CIÊNCIA DA

INFORMAÇÃO. 14., 2013. Florianópolis. [Paper...]. Florianópolis: UFSC, 2013.

Disponível em:

<http://enancib2013.ufsc.br/index.php/enancib2013/\%20XIVenancib/paper/viewFile/284/319

>. Acesso em: 13 jul. 2017.

SANTANA, Ricardo César Gonçalves; BONINI, Alfredo. Análise de dados sobre produção de leite: uma perspectiva da Ciência da Informação. Inf. \& Tecn. v. 1, n. 1, 2014.

Disponível em: <http://periodicos.ufpb.br/index.php/itec/article/view/19493/10863>. Acesso em: 21 jul. 2017.

SANTOS, Isabel Maria Francisca dos. Uma proposta de governança de dados baseada em um método de desenvolvimento de arquitetura empresarial. 2010. 140 p. Dissertação (Mestrado em Informática) - Centro de Ciências Exatas e Tecnologia, Universidade Federal do Rio de Janeiro, Rio de Janeiro, 2010. Disponível em:

<http://www2.uniriotec.br/ppgi/banco-de-dissertacoes-ppgi-unirio/ano-2010/uma-propostade-governanca-de-dados-baseada-em-um-metodo-de-desenvolvimento-de-arquiteturaempresarial/view>. Acesso em: 21 jul. 2017.

SILVA, Edna Lúcia da; MENEZES, Estera Muszkat. Metodologia da Pesquisa e Elaboração de Dissertação. 4. ed. Florianópolis: UFSC, 2005. Disponível em: $<$ https://projetos.inf.ufsc.br/arquivos/Metodologia_de_pesquisa_e_elaboracao_de_teses_e_di ssertacoes_4ed.pdf $>$. Acesso em: 20 jul. 2017.

SISTEMA de Avaliação Motora. [S.1. : s.n., 201-?]. Disponível em: <http://sam.motricidade.com.br/>. Acesso em: 27 jun. 2017.

SMITH, Michael L.; ERWIN, James. Role \& responsibility charting (RACI). In: Project Management Forum (PMForum). [2005]. Disponível em:

<https://pmicie.org/images/downloads/raci_r_web3_1.pdf>. Acesso em: 06 ago. 2017.

VIEIRA, Valter Afonso. As tipologias, variações e características da pesquisa de marketing. R. FAE, Curitiba, v. 5, n.1, p. 61-70, jan./abr. 2002. Disponível em: <https://revistafae.fae.edu/revistafae/article/view/449>. Acesso em: 24 jul. 2017.

WANG, Richard Y.; STRONG, Diane M. Beyond Accuracy: What Data Quality Means to Data Consumers. Journal of Management Information Systems, v. 12, n. 4, Spring 1996, p. 5-33. Disponível em:

<https://www.jstor.org/stable/40398176?seq=1\#page_scan_tab_contents >. Acesso em: 06 ago. 2017. 
WENDE, Kristin; OTTO, Boris. A contingency approach to data governance. In: INTERNATIONAL CONFERENCE ON INFORMATION QUALITY (IQ-2007), 12, 2007. Cambridge, USA. Research Paper... Cambridge, USA: Alexandria, 2007. Disponível em: <https://www.alexandria.unisg.ch/publications/213308>. Acesso em: 06 ago. 2017. 